\title{
Reflexión sobre el reconocimiento del contexto cultural en los procesos de enseñanza de la biología
}

\section{Reflection upon the cultural context recognition in biology teaching processes}

Por: Diana Pacheco Calderón*

Recibido: 25 -03- 2011

Aceptado: 06-06-2011

\section{Resumen}

La Licenciatura en Biología de la Universidad Pedagógica Nacional, en sus extensiones del Centro Valle de Tenza y los CERES de La Chorrera Amazonas y Puerto Asís - Puerto Caicedo Putumayo, propende por una enseñanza de la Biología en Contexto, que se constituye en un ejercicio participativo y de construcción colectiva entre los sabedores locales, estudiantes, indígenas, sabedores tradicionales, especialistas, docentes y comunidad, en torno a la comprensión de la vida y lo vivo, que ha permitido poner en diálogo los conocimientos disciplinares de occidente y los ancestrales de las comunidades, donde se propone construir un conocimiento biológico que responda tanto al plan de vida de las comunidades indígenas, como a las necesidades de las regiones, dado que Colombia es un país rico en diversidad biológica y cultural.

Palabras Claves: Enseñanza de la Biología, contexto, interculturalidad, construcción social de currículo, territorio.

\section{Summary}

The National Pedagogical University Biology degree, in its branches in Centro Valle de Tenza and La Chorrera Amazon basin and Puerto Asis - Putumayo Puerto Caicedo CERES foster for Biology teaching in context, which constitutes in a participatory and collective construction exercise among the local informed people, students, indigenous, traditional wise inhabitants, specialists, teachers and community about the understanding of life and living, which has allowed a dialogue about wisdom in the West and ancestral communities, which aims to build a biological knowledge that meets both the plan of life of indigenous communities and the needs of the regions, because Colombia is a rich country in biological and cultural diversity.

Keywords: Biology Teaching, context, interculturality, curriculum social construction, territory.

*Docente Investigadora de Planta Universidad Pedagógica Nacional. Licenciatura en BiologíaCentro Valle de Tenza. Correo electrónico: dpacheco@pedagogica.edu.co, dianapachecocalderon@yahoo.es 
"Mi consejo es que sigan inventando maneras de llegar a nuestra propia realidad, que entren en el detalle de las culturas. Nosotros como educadores debemos aprender mucho más de las realidades propias, saberlas distinguir, saberlas analizar, saberlas amar, y esas realidades están totalmente fuera de los esquemas educativos tradicionales y clásicos, que nos llevan a pensar por ejemplo, que son salvajes o bárbaros quienes viven en la Amazonia tropical, cuando por el contrario se está descubriendo que ellos son los poseedores del secreto de la supervivencia del mundo, por todo esto tenemos que aprender de ellos, de los indígenas, de los negros cimarrones, $y$ de los colonos...". Orlando Fals Borda.

El presente artículo, no es producto de una investigación, sino de un proceso reflexivo hacia la enseñanza de la Biología en contexto, desde la experiencia adelantada por la Universidad Pedagógica Nacional, en Colombia, en el cual respondiendo a uno de los propósitos contemplados en el Plan de Desarrollo Institucional, en el eje consolidación del carácter nacional, internacional e intercultural busca impulsar propuestas de educación que contribuyan a la consolidación de un país pluriétnico y multicultural, en el que se propicie el diálogo cultural de la distintas comunidades para proponer y orientar sus propios procesos educativos, ha venido propiciando a través de programas académicos en las regiones, un diálogo intercultural que lleve a la consolidación de un proyecto educativo, que permita nuevas maneras de pensar la educación y construir saber pedagógico desde la comprensión de los territorios en su diversidad.

Uno de los Departamentos que está liderando dicho proceso es Biología, cuya sede central se encuentra en Bogotá, y ofrece la Licenciatura en Biología en tres extensiones con modalidad presencial, en el Centro Valle de Tenza - Boyacá y los CERES ${ }^{1}$ de La Chorrera Amazonas y Puerto Asís- Puerto Caicedo Putumayo; los programas de formación de docentes se construyen socialmente de acuerdo a las necesidades de los contextos, a partir del reconocimiento de la interculturalidad, la diversidad y la inclusión social, los estudiantes pertenecen a comunidades campesinas mestizas; indígenas; colonos, mestizos y afrodescendientes, respectivamente. El programa fortalece el Proyecto Educativo Institucional, el cual propende por el carácter de nacionalización de la Universidad, así como a la necesidad de desarrollar un eje de reflexión acción sobre la educación intercultural.

Es así que la enseñanza de la Biología, en el proyecto curricular de la Licenciatura en sus extensiones se conciben desde una perspectiva de formación integral, que implica lo inter y transdisciplinar, de modo que se aporta a la formación desde los ámbitos pedagógicos, disciplinares, ambientales, socio-culturales, políticos y económicos; de

\footnotetext{
${ }^{1}$ Los Centros Regionales de Educación Superior CERES en Colombia, hacen parte de la política educativa del gobierno nacional para responder a las necesidades de educación superior en las regiones. Esta propuesta se centra en cuatro aspectos fundamentales: Ampliación de cobertura, equidad social en el acceso a la educación superior, mejoramiento tanto de la calidad educativa, como de la eficiencia del sector educativo.
} 
manera que se contemplan las diversas formas de construir conocimiento que tienen las comunidades, en el cual el currículo centra su mirada en el contexto.

Las experiencias adelantadas por el Departamento de Biología y sus extensiones, ha mostrado que es necesario que las disciplinas entren en diálogo con los saberes y las otras formas de concebir el mundo, igualmente que se reconozcan y se incluyan los principios que orientan los planes de vida ${ }^{2}$, lo cual se constituye en responde al contexto cultural. Para tal fin, actualmente se adelantan acciones educativas que contribuyen al docente en formación en su desarrollo cognitivo, intercultural, moral, ético, político, profesional docente, estético, lúdico, afectivo y comunicativo.

El proyecto curricular de la Licenciatura en Biología en las extensiones, construye socialmente los currículos con las comunidades con las que se tiene en cuenta, la pertinencia y relevancia del programa para el contexto biogeográfico y sociocultural, igualmente reflexiona en torno a las realidades históricas de las regiones, circunscritas en sus lógicas particulares, en sus procesos vitales de construcción como territorios y en el caso de la Amazonía de defensa; dado su carácter e importancia biogeográfica y geopolítica para la nación y el mundo; adicionalmente indaga sobre los desarrollos de las ciencias en el campo educativo a nivel local y regional, de modo que establece una interlocución de las múltiples expresiones culturales.

Desde esta perspectiva de construcción social e intercultural de los programas curriculares, se propone empoderar a los docentes en formación como conocedores de lo vivo, la naturaleza, la cultura y la biodiversidad de su territorio. De manera que se contribuye al fortalecimiento de la cultura en diálogo con otras culturas, mediante procesos de investigación y proyección social que permiten formar seres humanos autónomos, críticos, éticos, comprometidos con las comunidades y con el país desde el campo de la educación y de la Biología en particular; por lo anterior es importante que el licenciado en Biología trascienda no solo en lo disciplinar, sino como sujeto integral.

En consecuencia, la construcción social del proyecto curricular que lleva a cabo la Licenciatura en Biología, responde a las demandas socioculturales a nivel nacional y a la generación de estudios en interculturalidad tan necesarios en el contexto latinoamericano; entendiendo el enfoque intercultural según (Castaño, 2009) cuando nos reconocemos en y desde la diferencia, evitando anular las otras miradas. Esto significa valorar y legitimar los pensamientos y las prácticas distintas a las académicas

\footnotetext{
${ }^{2}$ En las comunidades indígenas de La Chorrera consideran: "El Plan de Vida es el plan que le entregó el padre creador al hombre, y donde según cada pueblo, contempla sus fundamentos, principios y normas de vida, es el plan que dice de dónde venimos, es el que nos da claridad de quiénes somos y es el que nos orienta para dónde vamos. Estos principios filosóficos que están en el plan son la base para interpretar el sentido y alcance de cada componente esencial y vital para los hijos del tabaco, la coca y la yuca dulce: población, territorio, gobierno, derecho propio y el plan de abundancia, que es donde vamos y la herramienta principal que nos permitirá en el futuro próximo seguir siendo pueblos.

Es la carta de navegación que permitirá amanecer la palabra en el Plan de Abundancia. Orienta la ruta que se debe seguir para atender las necesidades fundamentales de su población, territorio y gobierno propio. Es el soporte de la gestión de los proyectos que sí procurarán el compromiso estatal, gubernamental y no gubernamental en el departamento, la nación y la comunidad internacional, a través de mecanismos institucionales establecidos en el ordenamiento jurídico colombiano, tales como su articulación al Plan Departamental de Desarrollo y, por esta vía, al Plan Nacional de Desarrollo".
} 
convencionales, rompiendo con la idea de imponernos epistemológicamente a las culturas y grupos humanos definidos en la alteridad.

Esta perspectiva permite, el reconocimiento de las lógicas y formas en que las diversas comunidades habitan las regiones, construyen e interpretan su mundo desde donde es posible pensar en una educación que contribuye al desarrollo social, entendido como un proceso de crecimiento y cambio en el que las formas de organización, el sistema de relaciones en la comunidad, el bienestar común, y las dinámicas de enseñanza aprendizaje son ejes fundamentales.

Efecto espacial de los procesos organizativos, sino por el hecho de que cada región es el resultado de una historia en la que se ha ido configurando su cultura, identidad, el entorno institucional, económico y organizativo.

En la enseñanza de la Biología es importante partir del reconocimiento que los humanos somos seres situados en un espacio y tiempo determinado, permeados por una identidad y un territorio, que nos connotan en un grupo o sociedad específica, con personas concretas que nos rodean y con las cuales convivimos y creamos múltiples interrelaciones.

Es así, que es necesario establecer procesos dialógicos que recontextualicen y resignifiquen los saberes locales y se piense la enseñanza para cada contexto y la condición humana como resultado de procesos históricos, biológicos y culturales; como lo expresa (De Sousa, 2009) los limites y las posibilidades de cada conocimiento residen en la existencia de otros conocimientos y, por tanto, sólo pueden ser explorados y valorizados en comparación con otros conocimientos. De igual manera el descubrir nuestra naturaleza es tener conciencia para permitir que la armonía, equilibrio y vida se hagan realidad en cada uno de nosotros.

En cada humano reside una capacidad natural de autoreconocimiento para admirarnos, sorprendernos, y apasionarnos por las cosas; igualmente soñamos; tenemos inquietudes, gustos, inclinaciones, que debemos permitirnos descubrirlas para vivirlas a plenitud, y así dar paso al reconocimiento de los otros como seres legítimos y de esta manera, comenzar a crear y recrear didácticas propias, donde el eje central es la recuperación de los sujetos que comienzan a emerger en el reconocimiento de los contextos culturalmente diversos. Es importante tener en cuenta que occidente privilegia lo abstracto y entre los indígenas se privilegia el saber hacer, de modo que las didácticas es pertinente configurarlas hacia el saber hacer (Pacheco, 2010).

Como lo enuncié anteriormente, otros elementos transversales en la enseñanza de la Biología son la investigación y la proyección social, como procesos que permiten entender las interrelaciones de la naturaleza, la vida y lo vivo, con el entorno natural y social, además contribuye a propiciar el interés y la curiosidad, la capacidad crítica e indagadora en el estudiante; que lo conlleva a explorar y conceptualizar desde su propia experiencia a la integración y construcción de conocimientos para nuevos aprendizajes. 
En la Licenciatura en Biología en La Chorrera- Amazonas, con los docentes en formación indígenas, al interior del aula de clase se propicia una relación interétnica entre los Uitoto, Bora, Okaina, Muinane, Murui e Inga, por ejemplo, en los y escenarios tradicionales naturales como el río, la selva y la Chagra ${ }^{3}$; y con la maloka ${ }^{4}$ como escenario social, además se articulan los sitios sagrados, las formas de enseñanza tradicional indígena (oralidad), como también el docente en sus clases (Trujillo, 2010) da lugar para que los estudiantes preparen preguntas a las autoridades tradicionales que propicien y motiven las charlas nocturnas con docentes, estudiantes y miembros de la comunidad en la maloka.

Es decir vemos a la comunidad como sujetos de poder y de saber (reconocemos que ellos son portadores de un conocimiento y nosotros de otro, y ambos estamos, en ese sentido, en igualdad de condiciones). Esto hace que las relaciones ocupen otros lugares, y esto conlleva a un aprendizaje intercultural a partir del reconocimiento de lo propio, para ir a lo ajeno y volver a lo propio.

En ese sentido por ejemplo el espacio académico de organización biológica (Campos y Camero, 2009) brinda la oportunidad de comparar el modo de organización presentado por la Biología con el modo de organización presente en sus propias culturas. Durante este ejercicio de comparación, que se realiza entre los temas tratados en el aula correspondientes al pensamiento científico y los temas tratados en la maloka propios del saber ancestral, se evidencian algunas diferencias entre las formas de pensamiento occidental y ancestral con relación a la organización de la naturaleza. Ejemplos de estas diferencias se pueden ver en lo que cada forma de pensamiento considera acerca del

\footnotetext{
${ }^{3}$ Terreno de una o dos hectáreas, que se constituye en unidad familiar de transmisión de conocimiento cultural y producción de alimento, cuyas actividades están distribuidas entre el hombre y la mujer. En dicho espacio confluye lo espiritual y el trabajo físico.

4 "La maloka según la visión de la etnia Uitoto, es un microcosmos de los pueblos indígenas. La sostienen cuatro estantillos fuertes e igualmente importantes; sobre ella descansan las demás estructuras que la conforman. De la firmeza y la calidad de estos estantillos dependen asuntos relacionados con los procesos de maduración, protección, duración, seguridad y sabiduría. Cada estantillo representa cuatro elementos que a su vez son fortaleza: 1 . Un hijo del cacique y el cacique mismo. 2. Un baile. 3. Un color. 4. Una planta o animal.
}

Todo es de cuatro en cuatro; son cuatro los elementos con que moo buinaima creó el mundo: 1. Un pedazo de piedra; 2. un pedazo de palisangre; 3. un pedazo de hierro; 4. un poco de arena. Son cuatro los colores de los relámpagos: 1 . El verde, que anuncia que el tabaco, la coca y los frutos están creciendo; 2. el blanco, es el propio del moo buinaima o padre creador; 3. el rojo del tigre; 4. un poco amarillo, relámpago de la danza de la danta. Son cuatro los sabores Principales: 1. Estantillo de todas las frutas dulces; 2. Estantillo esencia de miel de abejas; 3. estantillo esencia del jugo de yuca dulce. 4. estantillo de todas las cosas dulces. Son cuatro los bailes principales: 1. Z+k+I; 2.yadiko; 3. Yuak+; 4. Meniza+. Son cuatro los olores de las esencias agradables: 1. Comejen; 2.Una flor; 3. Planta medicinal en forma de bejuco. 4. una hoja de comino. Son cuatro los dialectos del murui-m+n+ka: 1. M+ka; 2.M+n+ka; 3. N+pode; 4.Bue. Son cuatro los puntos cardinales 1. Oriente; 2. Occidente; 3. Norte; 4. Sur.

Entre los cuatro elementos hay equilibrio y armonía, no hay un elemento más importante que otro, los cuatro son igualmente fuerte. A partir de esta filosofía de la estructura de la maloka se empieza a concebir el proceso de enseñanza aprendizaje". "...Así mismo, el resultado que todo docente espera o toda institución espera es que sus alumnos sostengan la sociedad"d. Escrito elaborado en el espacio académico de Pedagogía y Didáctica I. Por José Jesús Zafiama Piñeros. Estudiante Universidad Pedagógica Nacional CERES La Chorrera Amazonas. 2010. 
carácter vivo o no vivo de muchos de los elementos de la naturaleza, de la influencia de la organización natural en la organización política (como se puede constatar en los papeles y funciones de diversos seres naturales en la organización social indígena por clanes), el carácter dinámico y cambiante frente al carácter estático de las identidades de los seres naturales, entre otros. De esta manera, el espacio brinda a los estudiantes una ocasión de reflexión sobre su cultura, en relación al pensamiento occidental representado por la Biología.

Es así como la educación propia de los pueblos indígenas se configura a partir de históricos) al interior de su cultura y de su dinámica endógena social.

Igualmente tomando como referente al mismo espacio académico en el Centro Valle de Tenza, con estudiantes de la región y provenientes de Bogotá ${ }^{5}$, la construcción de conocimiento sobre un criterio propio de organización biológica, se da basados en la teoría evolutiva y los conceptos básicos de ecología; de esta forma se espera que desde la concepción biológica en el contexto rural se estimule el reconocimiento de los saberes locales y la experiencia en torno a las explicaciones sobre la diversidad y organización de la vida. Por lo anterior, se contextualizó el concepto de organización biológica, desde los componentes histórico, cultural, político y económico y se consideró la organización biológica como una propiedad inherente de lo vivo (Pacheco; Campos, 2009).

Por consiguiente, el docente formado desde los presupuestos de ciencia y sociedad occidental que quiere aproximarse al mundo indígena debe tener especial cuidado al momento de interpretar el contexto, puesto que su mundo se rige por una cosmogonía y cosmovisiones en donde la relación ser humano-naturaleza se articula en un todo integrado: lo social se hace naturaleza y la naturaleza se hace social (naturaleza simbólica, cosmovisión, visión de territorio). Por lo anterior es necesario (Nuñez 2005) revalorar los saberes locales asociándolos a saberes universales, a través de prácticas educativas integradoras que garanticen la preservación de la biodiversidad natural y cultural.

De otro lado, los procesos de enseñanza de la Biología en comunidades desarrollada por docentes indígenas en su región posibilitará espacios en donde los lazos de confianza y afecto pueden ser elevados a su máxima expresión en la medida, en que el docente es un líder comunitario con amplio conocimiento de la naturaleza, (AZICATCH, 2007) ya que desde niño se formó en el reconocimiento de su entorno, aprendiendo a distinguir lo fundamental de la vida vegetal y animal y el respeto que se le debe como fuente de vida y adquieren el profundo sentido religioso con el cual se le cuida, viviendo en armonía con ella.

De esta manera la enseñanza de la Biología en contexto, con un enfoque intercultural da paso a colectividades epistémicas para la creación de espacios de inter-relaciones e interconocimientos, donde las comunidades rurales, urbanas e indígenas no pierdan su cultura, sino que se empoderen de sus territorios, y se creen puentes de comunicación entre el conocimiento científico, cotidiano y ancestral, que contribuyan al

\footnotetext{
${ }^{5}$ Estudiantes campesinos, y/o cuyas familias son de origen campesino y/o viven en sectores rurales.
} 
fortalecimiento de gobiernos propios y autónomos con entidad territorial; igualmente se aporte a la preservación de la naturaleza (agua, el suelo, oxígeno, fauna, y flora), en aras que se favorezca un desarrollo sustentable desde y para la región y se reconozca el hecho que habitamos en Colombia un país biodiverso, pluriétnico y multicultural.

Finalmente a través del presente artículo de reflexión, invito a que se continúen procesos investigativos alrededor de la creación de didácticas propias de la Biología en contextos culturalmente diversos, y asumir la interculturalidad como un proyecto político, donde se reconozcan a las comunidades como un legítimo otro.

\section{BIBLIOGRAFÍA}

Asociación zonal indígena de cabildos y autoridades tradicionales de la chorrera (AZICATCH). (2007). Plan de Vida de los hijos del tabaco, la coca y la yuca dulce y plan de abundancia de La Chorrera. Programa Amazónico, Codeba - Ecofondo y el auspicio de la Embajada del Reino de los Países Bajos. Colombia.

Campos, D; Camero, A. (2009). Informe espacio académico Organización Biológica, Licenciatura en Biología CERES La Chorrera. Trabajo no publicado. Universidad Pedagógica Nacional.

Castaño, N (2009) Construcción Social de Universidad para la inclusión: la formación de maestros con pertinencia y en contexto, desde una perspectiva intercultural. En Daniel Mato (coord.), Educación Superior, Colaboración Intercultural y Desarrollo Sostenible/Buen vivir. Experiencias en América Latina. Caracas: Instituto Internacional de la UNESCO para la Educación Superior en América Latina y el Caribe (IESALCUNESCO).

Dentro del baúl de un hombre caimán entrevista a Orlando Fals Borda (2007, Marzo), Magazín Pedagógico No 21.pp 4-5.Universidad Pedagógica Nacional.

De sousa, B. (2009). Una epistemología del sur. Siglo XX editores. México.

Nuñez, J. (2004). Pedagogía de los saberes campesinos, implicaciones para una educación rural. Caracas- Venezuela.

Pacheco, D; Campos, D. (2010). Programa de organización biológica, licenciatura en Biología. Trabajo no publicado. Universidad Pedagógica Nacional - Centro Valle de Tenza.

Pacheco, D. (2010). Informe final programa de educación rural e interculturalidad. Acompañamiento y consolidación de procesos de Interculturalidad VGU- CVT-01.09. Licenciatura en Biología - La Chorrera Amazonas. Trabajo no publicado. Universidad Pedagógica Nacional. 
Trujillo, M. (2010). Informe espacio académico Pedagogía y Didáctica II; primera parte. Licenciatura en Biología CERES La Chorrera. Trabajo no publicado. Universidad Pedagógica Nacional.

Universidad Pedagógica Nacional. (2009). Plan de Desarrollo Institucional 2009-2013. Una Universidad en permanente reflexión, innovación y consolidación. Colombia. 